\title{
Disfunção temporomandibular e sono: revisão integrativa de literatura
}

Temporomandibular dysfunction and sleep: integrative literature review

Disfunción temporomandibular y sueño: revisión integrativa de la literatura

Gabriele Ramos de LUCCAS $^{\mathbf{1}}$

Cléssia Eliene Simões de LIRA ${ }^{2}$

Keiliane Pereira GUEDES ${ }^{2}$

Lycia Wyara Oliveira SILVA ${ }^{2}$

José STECHMAN-NETO ${ }^{3}$

Camila de CASTRO CORRÊA ${ }^{4}$

${ }^{1}$ Doutoranda, Programa de Pós-Graduação em Fonoaudiologia, Faculdade de Odontologia de Bauru, Universidade de São Paulo (USP), 17012-901 Bauru, SP, Brasil

${ }^{2}$ Centro Universitário Planalto do Distrito Federal - UNIPLAN, 70297-400 Brasília-DF, Brasil

${ }^{3}$ Professor Adjunto, Universidade Tuiuti do Paraná 82010-330 Curitiba - PR, Brasil

\section{Resumo}

${ }^{4}$ Professora Titular do Curso de Fonoaudiologia, Centro Universitário Planalto do Distrito Federal - UNIPLAN, 70297-400 Brasília-DF, Brasil

Introdução: Estudos recentes apontam que existe associação entre qualidade do sono com disfunção temporomandibular (DTM), enfatizando que estas ambas alterações influenciam significantemente a qualidade de vida dos indivíduos. Objetivo: Investigar se há relação entre DTM e aspectos do sono. Método: O presente estudo foi realizado por meio de uma revisão integrativa da literatura referente às áreas de DTM e sono, especificando sobre qualidade do sono. A proposta foi realizada por meio de pesquisas na Lilacs, Pubmed, repositórios de teses, incluindo textos em português, espanhol e inglês, diretamente relacionados ao tema e sem limite nas datas de publicação. Resultados: Foram selecionados e analisados 19 artigos, cujos autores apontaram que a relação entre DTM e qualidade do sono, parâmetros polissonográficos, hábitos orais deletérios e queixas do sono. Os achados desses estudos mostraram que a má qualidade do sono pode estar relacionada à ansiedade, depressão e estresse, principalmente. Também não apresenta relação quanto os estágios do sono ou ao sexo dos participantes. Conclusão: observou-se que tanto para a população pediátrica quanto para a adulta, houve indícios de associação entre DTM e a ocorrência de bruxismo, alteração da qualidade do sono e dos parâmetros polissonográficos.

Descritores: Articulação Temporomandibular; Transtornos da Articulação Temporomandibular; Sono; Bruxismo do Sono; Fonoaudiologia.

\section{Abstract}

Introduction: Recent studies indicate that there is an association between sleep quality and temporomandibular disorders (TMD), emphasizing that these two changes significantly influence the quality of life of individuals. Objective: To investigate whether there is a relationship between TMD and aspects of sleep. Method: The present study was carried out through a bibliographic review referring to the areas of TMD and sleep, specifying on sleep quality. The proposal was carried out through research at Lilacs, Pubmed, repository of theses, including texts in Portuguese, Spanish and English, directly related to the topic and without limit on the publication dates. Results: 19 articles were selected and analyzed, whose authors pointed out that the relationship between TMD and sleep quality, polysomnographic parameters, harmful oral habits and sleep complaints. The findings of these studies showed that poor sleep quality may be mainly related to anxiety, depression and stress. It is also unrelated to the sleep stages or the sex of the participants. Conclusion: it was observed that for both the pediatric and adult population, there was evidence of an association between TMD and the occurrence of bruxism, changes in sleep quality and polysomnographic parameters.

Descriptors: Temporomandibular Joint; Temporomandibular Joint Disorders; Sleep; Sleep Bruxism; Speech, Language and Hearing Sciences.

\section{Resumen}

Introducción: Estudios recientes indican que existe una asociación entre la calidad del sueño y disfunción temporomandibular (DTM), enfatizando que estos dos cambios influyen significativamente en la calidad de vida de las personas. Objetivo: Investigar si existe una relación entre DTM y aspectos del sueño. Método: El presente estudio se realizó mediante revisión bibliográfica referida a las áreas de DTM y sueño, especificando la calidad del sueño. La propuesta se llevó a cabo a través de investigaciones en Lilacs, Pubmed, repositorio de tesis, incluyendo textos en portugués, español e inglés, directamente relacionados con el tema y sin límite en las fechas de publicación. Resultados: se seleccionaron y analizaron 19 artículos, cuyos autores señalaron la relación entre DTM y calidad del sueño, parámetros polisomnográficos, hábitos bucales nocivos y quejas del sueño. Los hallazgos de estos estudios mostraron que la mala calidad del sueño puede estar relacionada principalmente con la ansiedad, la depresión y el estrés. Tampoco está relacionado con las etapas del sueño o el sexo de los participantes. Conclusión: se observó que tanto para la población pediátrica como para la adulta, se evidenció asociación entre DTM y ocurrencia de bruxismo, cambios en la calidad del sueño y parámetros polisomnográficos.

Descriptores: Articulación Temporomandibular; Trastornos de la Articulación Temporomandibular; Sueño; Bruxismo del Sueño; Fonoaudiología.

INTRODUÇÃO

Em seu funcionamento normal, a articulação temporomandibular (ATM) realiza movimentos de abertura, lateralidade e protrusão da mandíbula, exerce papel fundamental na execução dos movimentos mandibulares e auxilia na manutenção do equilíbrio do sistema estomatognático e, portanto é considerada extremamente importante para o desempenho adequado das funções orofaciais ${ }^{1}$.

As Disfunções Temporomandibulares
(DTM) referem-se a um conjunto de alterações relacionadas a ATM, músculos da mastigação (temporal, masseter, pterigoideos medial e lateral) e estruturas associadas e, excetuandose as dores dentais, a DTM é considerada a principal fonte de dor na região orofacial. As DTMs possuem etiologia multifatorial, ou seja, são desencadeadas por diversos fatores que se relacionam entre si, sendo que, dentre eles, destacam-se fatores etiológicos intrínsecos (relacionados a patologias e más formações 
articulares e musculares), extrínsecos (traumas e acidentes externos) e problemas psicológicos ${ }^{2}$.

De acordo com os sintomas e sinais apresentados, as DTMs podem ser classificadas como transtornos dolorosos (mialgia local, dor miofascial com espalhamento e dor miofascial com dor referida, artralgia e cefaleias), articulares (deslocamento do disco com redução; com redução com bloqueio intermitente, sem redução sem limitação de abertura, com limitação de abertura e doença articular degenerativa) ou mistas3, podendo apresentar sintomas dolorosos e sinais de alteração articular ${ }^{4}$.

O sono consiste em uma função fisiológica fundamental para restabelecer energias, auxiliar no metabolismo e regular a produção de hormônios ${ }^{5}$. Os distúrbios do sono e as dificuldades para se manter uma boa qualidade desta função podem ocasionar sonolência excessiva diurna, irritabilidade, redução da qualidade de execução das atividades diárias e do nível de atenção e concentração, além de aumentar os riscos para acidentes laborais e de trânsito ${ }^{6}$.

A literatura aponta que a relação entre dor e sono é bidirecional, sendo a dor relacionada a fatores sensoriais, cognitivos e emocionais. A presença de distúrbios do sono em pacientes com DTM dolorosa é comum, sendo a queixa de má qualidade de sono o sintoma mais frequente. Em síntese, o sono de má qualidade é capaz de inibir a produção de neurotransmissores que são importantes para a modulação da dor e para a regulação do humor, estresse, ansiedade, sendo este fatores importantes para o controle da dor?

A relação entre sono e DTM tem sido objetivo de estudo de alguns autores, encorajados pela parcela significativa de indivíduos com DTM que se queixam de má qualidade do sono e, consequentemente, relatam prejuízos em sua vida diária ${ }^{7,8}$.

Portanto, considerando a relação entre dor e sono, e sabendo que a DTM pode estar relacionada a quadros de dor, o presente estudo teve como objetivo verificar se as DTMs relacionam-se com aspectos e distúrbios do sono. Espera-se que essa pesquisa traga contribuições teóricas importantes para a compreensão da relação entre qualidade do sono e DTM e, a partir dos resultados obtidos, espera-se que o fonoaudiólogo que atue com pacientes com DTM e/ou distúrbios do sono conscientize-se sobre a necessidade de investigar a relação entre estas duas condições nos indivíduos e realizar encaminhamentos e orientações assertivas, caso seja necessário.
MATERIAL E MÉTODO

O presente estudo foi realizado por meio de uma revisão integrativa da literatura referente a relação entre DTM e qualidade do sono. Para a pesquisa foi utilizado o cruzamento dos descritores "Disfunção Temporomandibular" AND "Sono", e seus respectivos correspondentes em inglês, "Temporomandibular Joint Disorders" AND "Sleep", nas seguintes bases de dados: Biblioteca Virtual em Saúde (BVS), Pubmed, Teses USP e Repositório UNESP.

Como critérios de inclusão, foram considerados os estudos que abordaram a relação entre DTM e sono, sendo excluídos os trabalhos sobre tipos de tratamento (placa de avanço, efeito de medicamentos, uso de CPAP) ou que incluíram na casuística comorbidades que poderiam agravar a DTM (fatores psicológicos, fibromialgia, artrite, artrose, Parkinson, Síndrome de Down), artigos de revisão de literatura e pesquisas que não estiveram disponíveis na íntegra pelo sistema VPN. A busca foi realizada por três pesquisadores, sem limitação temporal, e sem restrição de idioma.

Os estudos foram analisados e selecionados inicialmente a partir dos títulos e resumos para aplicação dos critérios de inclusão e exclusão e, após essa seleção prévia, os trabalhos foram lidos na íntegra para verificar se realmente eram adequados para análise.

RESULTADOS

A busca inicial nas bases de dados resultou em 878 trabalhos, sendo localizados 309 artigos na BVsalud, 544 na Pubmed, 17 no Tese USP e oito no Repositório UNESP. foram excluídos 782 trabalhos a partir da leitura do título e resumo por não se adequarem aos critérios da pesquisa, além de ser realizada a exclusão de outros 26 estudos por não estarem disponíveis na íntegra e 51 por serem repetições entre as bases. Portanto, foram selecionados 19 artigos, sendo que 0 fluxograma de busca é mostrado na Figura 1.

Para cada artigo selecionado foram relatadas as características relacionadas a base de dados, autores, ano, parâmetros de avaliação do sono e distúrbios do sono, objetivo principal, metodologia científica do trabalho e principais resultados e conclusão. Essas informações foram descritas no Quadro 1. Nota-se que a maior parte dos estudos evidenciou uma relação entre DTM e bruxismo do sono. 


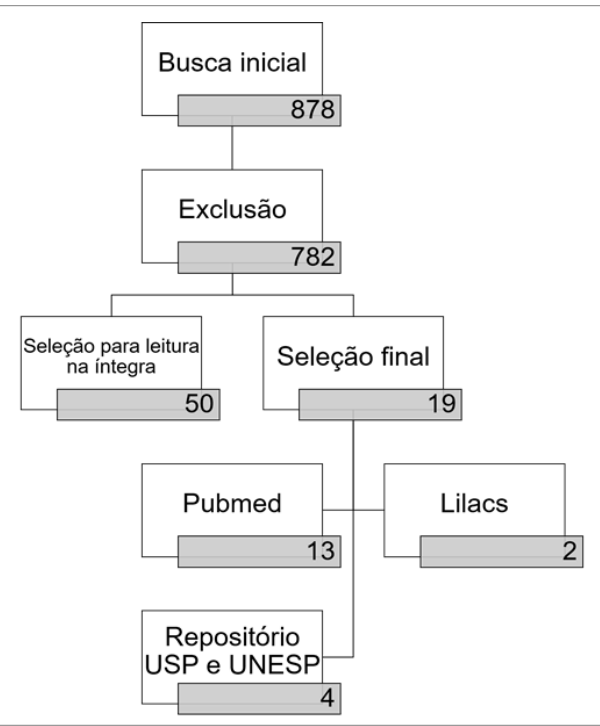

Figura 1: Fluxograma da busca de artigos nas bases de dados.

Quadro 1. Resultados da revisão bibliográfica quanto à alteração do sono, objetivo, métodos, resultados e conclusão

\begin{tabular}{|c|c|}
\hline \multicolumn{2}{|l|}{ Rossetti, 20069 } \\
\hline Base de Dados & Teses USP \\
\hline Parâmetros do Sono & $\begin{array}{l}\text { Bruxismo do sono } \\
\text { Parâmetros da PSG }\end{array}$ \\
\hline Objetivo & $\begin{array}{l}\text { Verificar a relacacão entre bruxismo do sono e dor miofascial, e } \\
\text { com a atividade rítmica dos músculos mastigatórios }\end{array}$ \\
\hline Métodos & $\begin{array}{l}30 \text { indivíduos com dor miofascial e } 30 \text { sem de DTM, que } \\
\text { relataram a intensidade de dor na face por meio de uma } \\
\text { escala visual analógica, responderam a um questionário de } \\
\text { DTM, um sobre bruxismo do sono, e foram submetidos a } \\
\text { exame físico de DTM e de bruxismo, além da PSG. }\end{array}$ \\
\hline $\begin{array}{l}\text { Resultados e } \\
\text { Conclusão }\end{array}$ & $\begin{array}{l}\text { Indivíduos do grupo com DTM relataram dor nos músculos } \\
\text { mastigatórios, além de apresentar relação com o bruxismo } \\
\text { do sono }\end{array}$ \\
\hline \multicolumn{2}{|r|}{ (2) } \\
\hline Base de Dados & Pubmed \\
\hline Parâmetros do Sono & $\begin{array}{l}\text { Bruxismo do sono } \\
\text { Parâmetros da PSG }\end{array}$ \\
\hline Objetivo & $\begin{array}{l}\text { Examinar a EMG durante o sono do músculo masseter na } \\
\text { DTM miofascial }\end{array}$ \\
\hline Métodos & $\begin{array}{l}124 \text { mulheres com DTM crônica, diagnosticadas pelo RDC e } \\
46 \text { mulheres controle pareadas, avaliadas pela } \\
\text { eletromiografia (bruxismo do sono) durante a PSG. OS } \\
\text { indivíduos com DTM classificaram a intensidade da dor de o } \\
\text { (sem dor) à 10 (pior dor). }\end{array}$ \\
\hline $\begin{array}{l}\text { Resultados e } \\
\text { Conclusão }\end{array}$ & $\begin{array}{l}\text { A EMG foi associada nos casos de DTM positivamente ao } \\
\text { bruxismo do sono e a intensidade da dor na viǵlia pós-sono }\end{array}$ \\
\hline \multicolumn{2}{|c|}{ Raphael et al., $2015^{11}$} \\
\hline Base de Dados & Pubmed \\
\hline Parâmetros do Sono & $\begin{array}{l}\text { Bruxismo do sono } \\
\text { Parâmetros da PSG }\end{array}$ \\
\hline Objetivo & $\begin{array}{l}\text { Verificar os autorrelatos de bruxismo do sono em indivíduos } \\
\text { com DTM. }\end{array}$ \\
\hline Métodos & $\begin{array}{l}124 \text { mulheres com DTM crônica e } 46 \text { mulheres sem DTM, } \\
\text { diagnóstico pelo RDC. Preencheram um questionário sobre } \\
\text { bruxismo do sono, realizaram dois exames de PSG } \\
\text { consecutivos e EMG dos músculos elevadores da mandíbula. }\end{array}$ \\
\hline $\begin{array}{l}\text { Resultados e } \\
\text { Conclusão }\end{array}$ & $\begin{array}{l}\text { O autorrelato previu ocorrência de dois ou mais episódios de } \\
\text { bruxismo durante o sono, mas não diferenciou o bruxismo } \\
\text { durante o sono moderado ou grave, comparando com a PSG. }\end{array}$ \\
\hline \multicolumn{2}{|c|}{ De Siqueira et al., $2017^{12}$} \\
\hline Base de Dados & Pubmed \\
\hline Parâmetros do Sono & Bruxismo do sono Parâmetros da PSG \\
\hline Objetivo & $\begin{array}{l}\text { Investigar se a presença de dor generalizada influencia } \\
\text { características polissonográficas de indivíduos com bruxismo } \\
\text { do sono e dor crônica nas ATMs }\end{array}$ \\
\hline Métodos & $\begin{array}{l}20 \text { indivíduos com bruxismo do sono e DTM (17 mulheres e } \\
3 \text { homens), divididos em a ausência (Grupo A) ou presença } \\
\text { (Grupo B) de dor generalizada. Os indivíduos foram } \\
\text { submetidos a avaliaçấo de dor orofacial (localização, } \\
\text { qualidade, duraçãa, tempo de agravamento da dor), presencaa } \\
\text { de dor de cabeça ou em outras regióes do corpo, além de } \\
\text { histórico médico. Além disso, foi aplicado o RDC e PSG para } \\
\text { confirmar a presença de bruxismo do sono, análise da } \\
\text { arquitetura do sono e presença de distúrbios do sono. }\end{array}$ \\
\hline $\begin{array}{l}\text { Resultados e } \\
\text { Conclusão }\end{array}$ & $\begin{array}{l}\text { O grupo B apresentou menor eficiência do sono e maior } \\
\text { média de idade, as queixas de dor orofacial autorreferidas, } \\
\text { aspectos clínicos e emocionais e os parâmetros } \\
\text { polissonográficos relacionados a bruxismo do sono foram } \\
\text { semelhantes nos dois grupos; todos os individuos } \\
\text { apresentaram dor miofascial mastigatória }\end{array}$ \\
\hline
\end{tabular}

Legenda: ATM - Articulação temparomadibular; AVM - Apertamento Voluntário Máximo; BS Bruxismo do Sono; CAS - Childres Anxiety Scale; CSHQ-PT - Childrens Sleep Habits Questionnaire DTM - Disfunção Temporomandibular; EMG - Eletromiografia; HADS - Escala Hospitalar de Ansiedade e Depressão; IMC - Índice de Massa Corporal; IAH - Índice de Apneia e Hipopneia; MAD Mecanismo de avanço mandibular; NRS - Numeric Rating Scales; PSG - Polissonografia; PSQI -
Índice de Qualidade do sono de Pittsburgh; RDC - Research Diagnostic Criteria; SAQ - Sleep
Quadro 1. (Continuação) Resultados da revisão bibliográfica quanto à alteração do sono, objetivo, métodos, resultados e conclusão

\begin{tabular}{|c|c|}
\hline \multicolumn{2}{|l|}{ Tay e Pang, $2018^{13}$} \\
\hline Base de Dados & Pubmed \\
\hline Parâmetros do Sono & Síndrome resistência das vias aéreas superiores/Bruxismo \\
\hline Objetivo & $\begin{array}{l}\text { Evidenciar as características clínicas e radiográficas de indivíduos } \\
\text { do Sudeste Asiático com DTM e com síndrome resistência das vias } \\
\text { aéreas superiores }\end{array}$ \\
\hline Métodos & $\begin{array}{l}86 \text { indivíduos ( } 26 \text { homens; } 60 \text { mulheres), apresentando queixa de } \\
\text { dor muscular orofacial persistente, submetidos a exame físico } \\
\text { completo, nasoendoscopia e teste noturno domiciliar de sono } \\
\text { usado no pulso. Responderam Escala de Sonolência de Epworth e } \\
\text { questionário de qualidade de vida }\end{array}$ \\
\hline $\begin{array}{l}\text { Resultados e } \\
\text { Conclusão }\end{array}$ & $\begin{array}{l}\text { A média do índice de despertar foi } 16,2 \text {, do IAH foi } 3,9 \text {, da Escala } \\
\text { de Sonolência de Epworth foi } 14,8 \text {. Observou } 66.3 \% \text { dores de } \\
\text { cabeça, } 41,9 \% \text { no pescoço, } 53,5 \% \text { na musculatura mastigatória } \\
68,6 \% \text { na ATM; } 90,7 \% \text { relataram bruxismo do sono, } 65,1 \% \text { com } \\
\text { deslocamento do côndilo na intercuspidação máxima com ou sem } \\
\text { estalo na ATM, postura de cabeça anteriorizada }(80.2 \%) \text { e } 91.9 \% \\
\text { congestionamento nasal. Concluiu-se possibilidade de relação da } \\
\text { DTM como resposta adaptativa à respiração inadequada durante } \\
\text { crescimento. }\end{array}$ \\
\hline \multicolumn{2}{|c|}{ Muzalev et al., $2020^{14}$} \\
\hline Base de Dados & Pubmed \\
\hline Parâmetros do Sono & Bruxismo do sono / Parâmetros da PSG \\
\hline Objetivo & Analisar o efeito da DTM dolorosa no bruxismo do sono \\
\hline Métodos & $\begin{array}{l}9 \text { homens com sinais clínicos de bruxismo do sono, submetidos a a } \\
\text { duas PSG, seguido por protocolo experimental de provocação da } \\
\text { dor. Após, foram submetidos a uma } 3^{\text {a }} \text { PSG para investigar o efeito } \\
\text { da dor no bruxismo do sono }\end{array}$ \\
\hline $\begin{array}{l}\text { Resultados e } \\
\text { Conclusão }\end{array}$ & $\begin{array}{l}\text { Foi observada redução de todos os parâmetros polissonográficos } \\
\text { para bruxismo entre } 3^{\mathrm{a}} \text { e } 2^{\mathrm{a}} \text { PSG.Dor induzida provocou } \\
\text { diminuição do bruxismo do sono. }\end{array}$ \\
\hline \multicolumn{2}{|l|}{ Fernandes, $2011^{15}$} \\
\hline Base de Dados & Repositório UNESP \\
\hline Parâmetros do Sono & Bruxismo do sono \\
\hline Objetivo & $\begin{array}{l}\text { Estimar o risco da ocorrência de DTM dolorosa e cefaleias } \\
\text { primárias em indivíduos com bruxismo do sono }\end{array}$ \\
\hline Métodos & $\begin{array}{l}301 \text { indivíduos ( } 253 \text { homens e } 48 \text { homens), divididos em: sem } \\
\text { DTM dolorosa e sem bruxismo do sono (15,9\%); indivíduos sem } \\
\text { DTM e com bruxismo do sono ( } 6,3 \% \text {; } 3 \text {; indivíduos com DTM } \\
\text { dolorosa e sem bruxismo do sono ( } 24,6 \% \text {; indivíduos com DTM } \\
\text { dolorosa e com bruxismo do sono (53,2\%). Foram avaliados por } \\
\text { meio do RDC, critérios clínicos para diagnóstico de bruxismo do } \\
\text { sono e da cefaleia. }\end{array}$ \\
\hline $\begin{array}{l}\text { Resultados e } \\
\text { Conclusão }\end{array}$ & $\begin{array}{l}\text { A prevalênnia de bruxismo do sono na amostra foi } 59,5 \% \text {; e para } \\
\text { cefaleia primária foi } 78,7 \% \text {; indivíduos com bruxismo do sono } \\
\text { apresentaram risco mais elevado para ocorrência de DTM } \\
\text { dolorosa; indivíduos com DTM e bruxismo do sono apresentaram } \\
\text { risco cinco vezes maior de apresentar dor miofascial; indivíduos } \\
\text { com DTM e com bruxismo do sono possuiam 12vezes mais } \\
\text { chances de apresentarem cefaleia. }\end{array}$ \\
\hline \multicolumn{2}{|l|}{ Costa, $2013^{16}$} \\
\hline Base de Dados & Teses USP \\
\hline Parâmetros do Sono & Bruxismo do sono \\
\hline Objetivo & $\begin{array}{l}\text { Analisar prevalência de bruxismo em crianças pré-escolares, } \\
\text { fatores relacionados e impacto na qualidade de vida }\end{array}$ \\
\hline Métodos & $\begin{array}{l}475 \text { pré-escolares ( } 4-5 \text { anos) foram submetidos ao exame clínico } \\
\text { intrabucal; responsáveis responderam questionário sobre } \\
\text { bruxismo e qualidade de vida relacionada a saúde oral. }\end{array}$ \\
\hline $\begin{array}{l}\text { Resultados e } \\
\text { Conclusão }\end{array}$ & $\begin{array}{l}\text { A prevalência de bruxismo na amostra foi } 47,4 \% \text {, sobressaliência } \\
\text { acentuada e hábitos roer unhas, morder lábios, mascar chicletes } \\
\text { respiração bucal relacionaram-se com maior prevalência de } \\
\text { bruxismo; sono agitado, dor de cabeça, crianças } \\
\text { agressivas/ansiosas/tímidas e com alteração na qualidade de vida } \\
\text { apresentaram maiores episódios }\end{array}$ \\
\hline \multicolumn{2}{|c|}{ Aguilera et al., $2014^{17}$} \\
\hline Base de Dados & Pubmed \\
\hline Parâmetros do Sono & Bruxismo do sono \\
\hline Objetivo & $\begin{array}{llllll}\begin{array}{l}\text { Analisar a combinação entre } \\
\text { características individuais na DTM }\end{array} & \text { sintomas } & \text { de } & \text { bruxismo } & \text { e } \\
\end{array}$ \\
\hline Métodos & $\begin{array}{l}\text { Foram avaliados } 1220 \text { indivíduos, com alterações muscular e } \\
\text { articular, de } 18 \text { a } 60 \text { anos, aplicação do RDC e questionário para o } \\
\text { diagnóstico de bruxismo do sono. }\end{array}$ \\
\hline $\begin{array}{l}\text { Resultados e } \\
\text { Conclusão }\end{array}$ & $\begin{array}{l}\text { A prevalência do bruxismo foi de } 54,51 \% \text { da amostra. Não foi } \\
\text { achado associação entre deslocamento de disco e o bruxismo. Fo } \\
\text { verificada forte associação entre relatos de bruxismo do sono e a } \\
\text { presença de dor devido à DTM, principalmente a DTM muscular } \\
\text { acompanhada de artralgia. }\end{array}$ \\
\hline \multicolumn{2}{|l|}{ Calderan, $2015^{18}$} \\
\hline Base de Dados & Teses USP \\
\hline Parâmetros do Sono & Bruxismo do sono \\
\hline Objetivo & $\begin{array}{l}\text { Analisar a relação do bruxismo do sono com ansiedade, estresse, } \\
\text { hábitos do sono, hábitos orais, força máxima de mordida } \\
\text { sinais/sintomas de DTM em crianças na faixa etária de } 6 \text { a } 8 \text { anos } \\
\text { de idade }\end{array}$ \\
\hline Métodos & $\begin{array}{l}43 \text { crianças, entre } 6 \text { a } 8 \text { anos, divididas em grupo sem e com } \\
\text { bruxismo. O diagnóstico de bruxismo foi realizado pela observação } \\
\text { de facetas de desgaste dentário, além de avaliação clinica } \\
\text { odontológica para DTM, força de mordida e condição oclusal. } \\
\text { Foram preenchidos os questionários: Escala de Stresse Infanti } \\
\text { aplicado na própria criança; Childrens Sleep Habits } \\
\text { Questionnaire- CSHQ-PT para investigar hábitos de sono da } \\
\text { criança a partir da percepção dos pais e Childrens Anxiety Scale- } \\
\text { SCAS-Brasil para analisar a percepção dos pais sobre ansiedade } \\
\text { das crianças. }\end{array}$ \\
\hline $\begin{array}{l}\text { Resultados e } \\
\text { Conclusão }\end{array}$ & $\begin{array}{l}\text { As crianças sem bruxismo do sono demonstraram maiores níveis } \\
\text { de ansiedade quando comparadas as crianças com bruxismo do } \\
\text { sono; não houve diferencas entre os grupos em relação aos } \\
\text { aspectos de estresse e hábitos do sono; todas as crianças com } \\
\text { bruxismo apresentaram pelo menos um tipo de hábito oral e } \\
\text { assimetria na força de mordida. }\end{array}$ \\
\hline
\end{tabular}
Legenda: ATM - Articulação temparomadibular; AVM - Apertamento Voluntário Máximo; BS Bruxismo do Sono; CAS - Childres Anxiety Scale; CSHQ-PT - Childrens Sleep Habits Questionnaire; DTM - Disfunção Temporomandibular; EMG - Eletromiografia; HADS - Escala Hospitalar de Ansiedade e Depressão; IMC - Indice de Massa Corporal; IAH - Indice de Apneia e Hipopneia; MAD Mecanismo de avanço mandibular; NRS - Numeric Rating Scales; PSG - Polissonografia; PSQI Índice de Qualidade do sono de Pittsburgh; RDC - Research Diagnostic Criteria; SAQ - Sleep Assessment Questionnaire. 
Quadro 1. (Continuação) Resultados da revisão bibliográfica quanto à alteração do sono, objetivo, métodos, resultados e conclusão

\begin{tabular}{|c|c|}
\hline \multicolumn{2}{|c|}{ Muzalev et al., $2017^{19}$} \\
\hline Base de Dados & Pubmed \\
\hline Parâmetros do Sono & Bruxismo do sono \\
\hline Objetivo & $\begin{array}{l}\text { Verificar os intervalos entre os eventos de bruxismo de indivíduos } \\
\text { come esm dor facial miofascial }\end{array}$ \\
\hline Métodos & $\begin{array}{l}86 \text { mulheres do grupo DTM e } 37 \mathrm{sem} \text { DTM, avaliacão pelo RDC, } \\
\text { PSG com pelo menos } 2 \text { episódios de bruxismo do sono por noite. }\end{array}$ \\
\hline $\begin{array}{l}\text { Resultados e } \\
\text { Conclusão }\end{array}$ & $\begin{array}{l}\text { A duracão dos intervalos interepisódio foi semellante nos grupos; } \\
\text { houve também um numero semelhante de episódios de bruxismo } \\
\text { do sono por hora e uma duracão total de episódios nos dois grupos }\end{array}$ \\
\hline \multicolumn{2}{|r|}{ 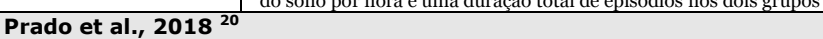 } \\
\hline Base de Dados & Pubmed \\
\hline Parâmetros do Sono & Bruxismo do sono \\
\hline Objetivo & $\begin{array}{l}\text { Investigar o bruxismo do sono e a associacão com o sono, uso de } \\
\text { aparelho ortodôntico e sinais de alteração do sistema } \\
\text { estomatognático em adolescentes }\end{array}$ \\
\hline Métodos & $\begin{array}{l}231 \text { adolescentes, } 12 \text { anos de idade, e seus responsáveis, que } \\
\text { responderam um questionário sobre o sono, realizacáo de } \\
\text { tratamento ortodôntico com aparelhos fixos, exame extraoral e } \\
\text { intraoral para identificar alteraçâes do sistema estomatognatico. }\end{array}$ \\
\hline $\begin{array}{l}\text { Resultados e } \\
\text { Conclusão }\end{array}$ & $\begin{array}{l}\text { Prevalência de bruxismo do sono foi } 16,9 \% \text {, aumentada quando } \\
\text { associado ao ronco e uso de aparelhos ortodônticos. }\end{array}$ \\
\hline \multicolumn{2}{|c|}{ Schmitter et al., $2019^{21}$} \\
\hline Base de Dados & Pubmed \\
\hline Parâmetros do Sono & Bruxismo do sono \\
\hline Objetivo & $\begin{array}{l}\text { Analisar a correlacão entre estresse crônico e atividade da } \\
\text { musculatura temporal durante a noite }\end{array}$ \\
\hline Métodos & $\begin{array}{l}44 \text { mulheres, com e sem queixa de bruxismo do sono, dor crônica, } \\
\text { responderam questionários sobre historia clinica, presença de } \\
\text { bruxismo do sono, HADS, escala para avaliaçao do estresse e } \\
\text { eletromiografia noturna para avaliar atividade músculo temporal. }\end{array}$ \\
\hline $\begin{array}{l}\text { Resultados e } \\
\text { Conclusão }\end{array}$ & $\begin{array}{l}\text { Os indivíduos com DTM apresentaram correlacãão do HADS } \\
\text { ("sobrecarga de trabalho" e "pressão para executar" - referentes } \\
\text { ao trabalho) com atividade do músculo temporal durante o sono }\end{array}$ \\
\hline \multicolumn{2}{|l|}{ Martins et al., $2010^{2}$} \\
\hline Base de Dados & Pubmed \\
\hline Parâmetros do Sono & Qualidade do sono \\
\hline Objetivo & $\begin{array}{l}\text { Investigar a associacãão entre qualidade do sono e estresse em } \\
\text { indivíduos com DTM }\end{array}$ \\
\hline Métodos & $\begin{array}{l}354 \text { adultos, submetidos ao Questionário da Fonseca (registrar } \\
\text { gravidade da DTM),PSQI ( avaliar a qualidade do sono) e Escala } \\
\text { de Avaliaçấo do Reajuste Social (registrar nivel de estresse) }\end{array}$ \\
\hline $\begin{array}{l}\text { Resultados e } \\
\text { Conclusão }\end{array}$ & $\begin{array}{l}\text { 50,8\% com DTM, correlacionando com o estresse e a presença de } \\
\text { alteraçẫo da qualidade do sono }\end{array}$ \\
\hline \multicolumn{2}{|c|}{ Oliveira et al., $2015^{23}$} \\
\hline Base de Dados & Pubmed \\
\hline Parâmetros & Qualidade do sono \\
\hline Objetivo & $\begin{array}{l}\text { Avaliar a DTM, a ansiedade, a qualidade do sono e de vida em } \\
\text { enfermeiros de Uberlândia. }\end{array}$ \\
\hline Métodos & $\begin{array}{l}160 \text { enfermeiros responderam a quatro questionários } \\
\text { (questionario de Fonseca para avaliar DTM, IDATE ansiedade, } \\
\text { SAQ qualidade do sono, SF-36 qualidade de vida). }\end{array}$ \\
\hline $\begin{array}{l}\text { Resultados e } \\
\text { Conclusão }\end{array}$ & $\begin{array}{l}41 \text { enfermeiros sem DTM, } 66 \text { com DTM leve, } 39 \text { DTM moderada e } \\
14 \text { com DTM grave. A DTM esteve associada à ansiedade, a } \\
\text { gravidade da DTM se relacionou à gravidade da ansiedade, leve, } \\
\text { moderada e grave. A presença de DTM pode diminuir a qualidade } \\
\text { do sono e a de vida. }\end{array}$ \\
\hline \multicolumn{2}{|r|}{ do sono e a de vida. } \\
\hline Base de Dado & Lilacs \\
\hline Parâmetros do Sono & Qualidade do sono \\
\hline Objetivo & $\begin{array}{l}\text { Avaliar o limiar de dor, qualidade do sono e níveis de ansiedade } \\
\text { em participantes com diferentes tipos de DTM }\end{array}$ \\
\hline Métodos & 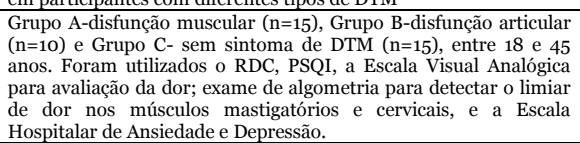 \\
\hline $\begin{array}{l}\text { Resultados e } \\
\text { Conclusão }\end{array}$ & $\begin{array}{l}\text { Os grupos A e B mostraram prejuízos na qualidade do sono, níveis } \\
\text { de ansiedade, menor valor de limiar de dor nos músculos } \\
\text { mastigatórios e trapézio quando comparados ao controle. }\end{array}$ \\
\hline \multicolumn{2}{|l|}{ Natu et al., $2018^{25}$} \\
\hline Base de Dados & Pubmed \\
\hline Parâmetros do Sono & Qualidade do sono \\
\hline Objetivo & $\begin{array}{l}\text { Investigar presenca de DTM e relacões com qualidade de vida, } \\
\text { depressão,ansiedade e qualidade do sono (jovens Sudeste Asiático) }\end{array}$ \\
\hline Métodos & $\begin{array}{l}244 \text { alunos de uma politécnica ( } 37 \text { homens, } 207 \text { mulheres, idade } \\
\text { média de 20,1 anos) que responderam a um questionario para } \\
\text { analisar a gravidade da DTM (Fonesca's Anamnestic Index), } \\
\text { qualidade de vida na saúde oral (Oral Health Impact Profile for } \\
\text { TMD - OHIP-TMD), escalas para investigar depressão e } \\
\text { ansiedade (DASS-21) e o PSQI (qualidade do sono). }\end{array}$ \\
\hline $\begin{array}{l}\text { Resultados e } \\
\text { Conclusão }\end{array}$ & $\begin{array}{l}32,4 \% \text { apresentaram DTM leve, } 9,4 \% \text { tiveram DTM moderada e } \\
58,2 \% \text { não obtiveram escore para DTM. A prevalência total de } \\
\text { DTM foó } 41,8 \% \text { e a qualidade de sono, de vida na saúde oral, } \\
\text { depressão e ansiedade variaram de acordo com gravidade da DTMM. }\end{array}$ \\
\hline \multicolumn{2}{|c|}{ Dubrovsky et al., $2017^{26}$} \\
\hline Base de Dados & Pubmed \\
\hline Parâmetros do Sono & Qualidade do sono / Parâmetros da PSG \\
\hline Objetivo & $\begin{array}{l}\text { Avaliar como questionários de qualidade do sono variam em } \\
\text { funcão da intensidade da dor, PSG e sintomas depressivos em } \\
\text { mulheres com DTM e controles pareados demograficamente }\end{array}$ \\
\hline Métodos & 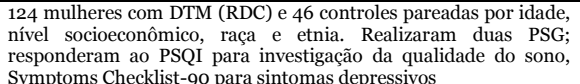 \\
\hline $\begin{array}{l}\text { Resultados e } \\
\text { Conclusão }\end{array}$ & $\begin{array}{l}\text { Os casos de DTM apresentaram escores piores no PSQI e mais } \\
\text { sintomas depressivos. A PSG demonstrou maior latenciaia para o } \\
\text { sono REM nos casos de DTM e mais despertares em todos os } \\
\text { participantes. Ador não se relaciononou ao PSQI. }\end{array}$ \\
\hline & $\begin{array}{l}\text { MAD } \\
\text { MSQI } \\
\text { PSQ }\end{array}$ \\
\hline
\end{tabular}

Quadro 1. (Continuação) Resultados da revisão bibliográfica quanto à alteração do sono, objetivo, métodos, resultados e conclusão

\begin{tabular}{|c|c|}
\hline \multicolumn{2}{|c|}{ Ribeiro et al., $2016^{27}$} \\
\hline Base de Dados & Lilacs \\
\hline Parâmetros do Sono & Queixas de sono \\
\hline Objetivo & $\begin{array}{l}\text { Relacionar as adaptações craniofaciais e da ATM à qualidade } \\
\text { de vida em respiradores orais }\end{array}$ \\
\hline Métodos & $\begin{array}{l}73 \text { escolares de } 7 \text { à } 14 \text { anos, com respiração oral, } \\
\text { apresentando alterações craniofaciais e agrupados de acordo } \\
\text { com o sexo (feminino n= } 40 / \text { masculino } n=33 \text { ) e por idade }(< \\
10 \text { anos } n=29 />10 \text { anos } n=44) \text {. Foram avaliados por } \\
\text { fisioterapeutas em relação aos movimentos mandibulares e } \\
\text { postura craniocervical; responderam a um questionário } \\
\text { sobre a alimentação, escolaridade e queixas de sono }\end{array}$ \\
\hline $\begin{array}{l}\text { Resultados e } \\
\text { Conclusão }\end{array}$ & $\begin{array}{l}\text { A presença de desvio em abertura de boca e em protrusão } \\
\text { relacionou-se com a presença de boca seca ao acordar, } \\
\text { alimentação, escolaridade e sono. Escolares com idade } \\
\text { superior a 10 anos apresentaram mais queixas de sono } \\
\text { durante o dia; escolares com idade superior a } 10 \text { anos } \\
\text { apresentaram mais dificuldades em aprender quando } \\
\text { comparados ao grupo com idade inferior }\end{array}$ \\
\hline
\end{tabular}

Legenda: ATM - Articulação temparomadibular; AVM - Apertamento Voluntário Máximo; BS Bruxismo do Sono; CAS - Childres Anxiety Scale; CSHQ-PT - Childrens Sleep Habits Questionnair Ans - Mecanismo de avanço mandibular; NRS - Numeric Rating Scales; PSG - Polissonografia; PSO Índice de Qualidade do sono de Pittsburgh; RDC - Research Diagnostic Criteria; SAQ - Sleep Assessment Questionnaire.

\section{DISCUSSÃO}

A revisão de literatura teve como objetivo investigar a relação entre DTM e sono. Após a busca nas bases de dados e aplicação dos critérios de inclusão e exclusão, foram considerados 19 trabalhos para análise ${ }^{9-27}$, os quais demonstraram possíveis correlações da DTM com o bruxismo do sono, parâmetros da PSG, alteração da qualidade do sono e sonolência diurna excessiva. O período de publicação das pesquisas foi entre 2003 e 2020, sendo que a maioria ocorreu nos últimos cinco anos, demonstrando que a temática abordada nesta revisão tem ganhado espaço no decorrer dos anos. Ainda, os resultados evidenciam que - Brasil é um dos países que mais tem publicado sobre esse assunto, demonstrando que o país tem interesse em realizar avanços científicos nessa área.

Dos 19 trabalhos selecionados, 14 abordaram a relação entre DTM e bruxismo do sono, indicando a presença da associação entre esses dois distúrbios e evidenciando os prejuízos na qualidade e eficiência do sono em indivíduos com DTM dolorosa. Sendo a DTM uma disfunção de causas multifatoriais, o bruxismo do sono é um dos fatores que podem colaborar para o surgimento ou agravamento de um quadro de DTM, já que o ranger dos dentes pode ocasionar desequilíbrios oclusais, musculares e funcionais que podem desencadear ou agravar quadros de dor. Além disso, é importante lembrar que o bruxismo do sono está relacionado à piora da qualidade do sono, o que por si só já seria capaz de comprometer a modulação e controle da dor e, portanto, relacionar-se com os sintomas da DTM.

A investigação de parâmetros polissonográficos foi realizada em alguns desses estudos, constatando uma relação entre 
DTM e dor na musculatura mastigatória e bruxismo do sono ${ }^{9-11}$. A literatura aponta que a musculatura mastigatória em indivíduos com bruxismo do sono realiza ações e demandas atípicas, podendo ocasionar alterações neuromusculares capazes de desencadear dor, cansaço e fadiga nesses indivíduos, com possíveis impactos no desempenho da função mastigatória ${ }^{28}$. Outro estudo evidenciou a presença de sonolência diurna excessiva em indivíduos com dor orofacial e com queixas de bruxismo do sono ${ }^{13}$, sendo esse um sintoma comum (sonolência) em indivíduos adultos com possíveis distúrbios do sono ${ }^{29}$. Por fim, um estudo não encontrou diferença entre indivíduos com e sem dor em relação a frequência e duração dos intervalos entre episódios de bruxismo do sono ${ }^{19}$, enquanto que os mesmos autores, em uma publicação recente, evidenciaram que o aumento da dor muscular induzida diminuiu a ocorrência dos episódios de bruxismo do sono ${ }^{14}$, apontando uma relação negativa entre dor e episódios de bruxismo, possivelmente relacionada a questões de adaptação a dor?. A discordância entre os estudos pode ser explicada por limitações do equipamento de registro, que não possuem gravação de áudio e vídeo, o que dificulta a exclusão de artefatos relacionados a outras atividades motoras orais. Pesquisas futuras com equipamentos com gravação de áudio e vídeo são importantes para compreender os resultados.

Ainda sobre os 14 estudos sobre
bruxismo do sono, notou-se um
comprometimento dos autores em utilizar
exames padrão ouro para diagnóstico das condições estudadas, a exemplo de estudos que analisaram o sono por polissonografia e a DTM pelo exame RDC ${ }^{9-12,14,19}$. Além disso, também utilizaram questionários para analisar as questões relacionados ao sono, sintomas de DTM e qualidade de vida ${ }^{13,16,23,25,27}$. O uso de exames padrão ouro para diagnóstico é importante para maior confiabilidade dos resultados e comparação com estudos prévios, enquanto que a aplicação de questionário auxilia na compreensão dos sintomas apresentados pelos indivíduos dos estudos.

Os seis trabalhos que abordaram 0 bruxismo do sono por meio de questionários, de forma exclusiva, constataram alta ocorrência do bruxismo do sono na população com DTM ${ }^{15,17,21}$, além da associação com cefaléia ${ }^{15,16}$, mialgia em elevadores de mandíbula ${ }^{15}$, hábitos orais $^{16,18}$, respiração oral ${ }^{16}$, sono agitado $^{16}$, alteração do temperamento ${ }^{16,18}$, alteração na qualidade de vida ${ }^{16}$ e influência do ronco e uso de aparelhos ortodônticos no bruxismo do sono em adolescentes ${ }^{20}$. Esses dados evidenciam que os indivíduos com DTM apresentam sintomas dolorosos e relacionados a aspectos do sono.

Diante do exposto, observa-se que há divergência entre os estudos em relação à associação de DTM e bruxismo do sono, embora os resultados encontrados evidenciem uma possível associação. Dentre os fatores que podem explicar a heterogeneidade dos resultados, pode-se citar a diversidade entre os grupos de estudo com e sem DTM, métodos de avaliação da DTM (não utilização sistemática do $\mathrm{RDC}$ ), faixas etárias abordadas e delineamento do estudo, sendo que a maioria propôs avaliações em períodos curtos de tempo. Estudos com delineamentos capazes de investigar a relação de causa e efeito entre duas condições, tais como pesquisas de coorte, serão importantes no futuro para compreender melhor esta associação.

Da amostra de estudos selecionados, cinco trabalhos investigaram a qualidade de sono, demonstrando uma relação com a DTM $^{22-26}$, além de associação com estresse ${ }^{22}$ e ansiedade $^{23,25}$. A relação entre DTM e fatores como ansiedade e estresse é bem estabelecida na literatura ${ }^{30}$.

Ressalta-se que a duração do tempo de sono ou sua interrupção não foram medidas nas pesquisas para que se pudesse ter a exata noção de quantas horas do tempo básico de sono (8 horas) são perdidos com insônia ou despertar frequente, demonstrando limitações metodológicas nos estudos abordados. Além disso, conforme citado pelos próprios autores, o número e composição das amostras prejudicaram determinadas análises, sendo necessários estudos com grupos maiores de participantes e mais homogêneos em suas características. Observa-se que relação entre DTM, qualidade do sono e ansiedade foi abordada com frequência pelos autores, evidenciando que quanto maior a intensidade da DTM dolorosa, maior a ansiedade apresentada pelos pacientes e, consequentemente, com alterações na qualidade do sono.

Houve predominância das investigações na população adulta, entretanto três estudos investigaram aspectos do sono e DTM em crianças e adolescentes. Constatou-se que escolares com bruxismo do sono apresentaram associação com respiração oral, desvio em abertura de boca e em protrusão, boca seca ao acordar, mais dificuldades em aprender ${ }^{27}$, hábitos orais deletérios, assimetria na força de mordida $^{18}$, sono agitado, dor de cabeça e 
alteração do temperamento ${ }^{16}$. Estudos sobre DTM e distúrbios do sono com foco na população infantil são necessários, considerando a importância da intervenção precoce para diminuir a probabilidade de comorbidades, e o papel do sono no crescimento craniofacial, desenvolvimento infantil e desempenho escolar ${ }^{31-34}$.

Houve predominância da casuística de mulheres nos estudos sobre o bruxismo do sono, impossibilitando realizar a relação dos distúrbios com 0 sexo. Os achados demonstraram relação do bruxismo do sono, com DTM, cefaleia, alterações físicas, depressão e ansiedade. Ainda reforçaram que os questionários de autoavaliação/autorrelato não apresentam acurácia adequada para 0 diagnóstico do bruxismo do sono ${ }^{11}$, corroborando com consenso da área, que estabelecem a indicação da polissonografia para este diagnóstico ${ }^{35}$.

Por fim, ressalta-se ainda a necessidade de atenção por parte dos fonoaudiólogos à temática apresentada, visto que os principais pesquisadores dos trabalhos são da área de Odontologia. A Fonoaudiologia tem se consolidado nas áreas de DTM e distúrbios do sono, sendo essencial estabelecer vínculos entre as duas temáticas para proporcionar ao paciente 0 melhor tratamento possível. Ao compreender a associação entre as duas condições é possível investigar possíveis sinais e sintomas, realizar encaminhamentos e estabelecer metas terapêuticas mais assertivas.

CONCLUSÃO

Desta forma, observou-se que tanto para a população pediátrica quanto para a adulta, houve indícios de associação entre DTM e a ocorrência de bruxismo, alteração da qualidade do sono e dos parâmetros polissonográficos.

\section{REFERÊNCIAS}

1. Sassi FC, Silva AP, Santos RKS, Andrade CRF. Tratamento para disfunções temporomandibulares: uma revisão sistemática. Audiol Commun Res. 2018;23:1-13.

2. De Rossi SS, Greenberg MS, Liu F, Steinkeler A. Temporomandibular disorders: evaluation and management. Med Clin North Am. 2014;98(6):1353-84.

3. Pereira Júnior FJ, Gonçalves DAG. Critérios de Diagnóstico para Desordens Temporomandibulares: Protocolo Clínico e Instrumentos de Avaliação Brazilian Portuguese [Internet]. 2020. Available from: https://ubwp.buffalo.edu/rdc-

tmdinternational/tmd-assessmentdiagnosis/dctmd/dc-tmd-translations/

4. Chantaracherd P, John MT, Hodges JS,
Schiffman EL. Temporomandibular joint disorders' impact on pain, function, and disability. J Dent Res. 2015;94(3 Suppl):79S-86S.

5. Troynikov O, Watson CG, Nawaz N. Sleep environments and sleep physiology: A review. J Therm Biol. 2018;78:192-203.

6. Ramar K, Olson EJ. Management of common sleep disorders. Am Fam Physician. 2013;88(4):231-8

7. Almoznino G, Benoliel R, Sharav $Y$, Haviv $Y$. Sleep disorders and chronic craniofacial pain: Characteristics and management possibilities. Sleep Med Rev. 2017;33:39-50.

8. Mathias JL, Cant ML, Burke ALJ. Sleep disturbances and sleep disorders in adults living with chronic pain: a meta-analysis. Sleep Med. 2018;52:198-210.

9. Rosseti LMON. Associação entre bruxismo do sono e dor miofascial: um estudo polissonográfico [tese]. Bauru; Universidade de São Paulo (USP); 2006.

10. Raphael KG, Janal MN, Sirois DA, Dubrovsky $B$, Wigren PE, Klausner JJ et al. Masticatory muscle sleep background EMG activity is elevated in myofascial TMD patients. J Oral Rehabil 2013;40(12):883-91.

11. Raphael KG, Janal MN, Sirois DA, Dubrovsky $B$, Klausner JJ, Krieger AC et al. Validity of selfreported sleep bruxism among myofascial temporomandibular disorder patients and controls. J Oral Rehabil. 2015;42(10):751-58.

12. Siqueira JTT, Camparis CM, Siqueira SRDT, Teixeira MJ, Bittencourt L, Tufik S. Effects of localized versus widespread TMD pain on sleep parameters in patients with bruxism: a singlenight polysomnographic study. Arch Oral Biol. 2017;76:36-41.

13. Tay DKL, Pang KP. Clinical phenotype of South East Asian temporomandibular disorder patients with upper airway resistance syndrome. J Oral Rehabil. 2018;45:25-33.

14. Muzalev K, Visscher CM, Koutris M, Lobbezoo F. Effect of experimental temporomandibular disorder pain on sleep bruxism: a pilot study in males. Clin Oral Investig. 2020;24(1):103-11.

15. Fernandes G. Estudo da associação entre bruxismo do sono, disfunção temporomandibular e cefaleias primárias [dissertação]. Araraquara: Universidade Estadual Paulista "Júlio de Mesquita Filho", Araraquara; 2011.

16. Costa SV. Bruxismo na infância: estudo clínico aleatório sobre fatores relacionados à ocorrência e influência na qualidade de vida [dissertação]. Bauru: Universidade de São Paulo (USP); 2013.

17. Aguilera AB, Lopez LG, Aguilera EB, Aizpurua JLDLH, Torronteras AR, Saint-Gerons RS et al. Relationship between self-reported sleep bruxism and pain in patients with temporomandibular disorders. J Oral 
Rehabil.2014;41:564-72.

18. Calderan MF. Hábitos do sono, estresse e ansiedade de crianças com bruxismo [dissertação]. Bauru: Universidade de São Paulo; 2015.

19. Muzalev K, Lobbezoo F, Janal MN, Raphael KG. Interepisode sleep bruxism intervals and myofascial face pain. Rev Sleep. 2017;40(8):16.

20. Prado IM, Abreu LG, Silveira KS, Auad SM, Paiva SM, Manfredini D et al. Study of associated factors with probable sleep bruxism among adolescents. J Clin Sleep Med. 2018;14(8):1369-76.

21. Schmitter $M$, Kares-Vrincianu $A$, Kares $H$, Malsch C, Schindler HJ. Chronic stress and temporalis muscle activity in TMD patients and controls during sleep: a pilot study in females. Clin Oral Investig. 2019;23:667-72.

22. Martins RJ, Garbin CAS, Garcia AR, Garbin AJI, Miguel N. Stress levels and quality of sleep in subjects with temporomandibular joint dysfunction. Rev odonto ciênc. 2010;25(1):326.

23. Oliveira LK, Almeida GA, Lelis ÉR, Tavares M, Fernandes Neto AJ. Temporomandibular disorder and anxiety, quality of sleep, and quality of life in nursing professionals. Braz Oral Res. 2015;29(1):1-7.

24. Daher CRM, Cunha LF, Ferreira APL, Souza AISO, Rêgo TAM, Araújo MGR et al. Limiar de dor, qualidade do sono e níveis de ansiedade em indivíduos com disfunção temporomandibular. Rev CEFAC. 2018;20(4):450-58.

25. Natu VP, Yap AU-J, Su MH, Ali MNI, Ansari A. Temporomandibular disorder symptoms and their association with quality of life, emotional states and sleep quality in SouthEast Asian youths. J Oral Rehabil. 2018;45:756-63.

26. Dubrovsky B, Janal MN, Lavigne GJ, Sirois DA, Wigren PE, Nemelivsky L, Krieger AC et al. Depressive symptoms account for differences between self-reported versus polysomnographic assessment of sleep quality in women with myofascial TMD. J Oral Rehabil. 2017;44;925-33.

27. Ribeiro AF, Morosini LM, Nathalia Silveira Finck NS, Pacheco MCT, Araújo MTM. Associação entre as adaptações da articulação temporomandibular e a qualidade de vida de escolares respiradores bucais. Rev Fisiot Bras. 2016;17(4):1-11.

28. Ferreira MC, Porto de Toledo I, Dutra KL, Stefani FM, Porporatti AL, Flores-Mir C, De Luca Canto G. Association between chewing dysfunctions and temporomandibular disorders: A systematic review. J Oral Rehabil. 2018;45(10):819-35.
29. Hurlston A, Foster SN, Creamer J, Brock MS, Matsangas P, Moore BA, Mysliwiec V. The Epworth Sleepiness Scale in Service Members with Sleep Disorders. Mil Med. 2019;184(1112):e701-7.

30. Kobayashi FY, Gavião MBD, Marquezin MCS, Fonseca FLA, Montes ABM, Barbosa TS, Castelo PM. Salivary stress biomarkers and anxiety symptoms in children with and without temporomandibular disorders. Braz Oral Res. 2017;31:e78.

31.Perez C. Temporomandibular disorders in children and adolescents. Gen Dent. 2018;66(6):51-5.

32. Christidis N, Lindström Ndanshau E, Sandberg A, Tsilingaridis G. Prevalence and treatment strategies regarding temporomandibular disorders in children and adolescents-A systematic review. J Oral Rehabil. 2019;46(3):291-301.

33. Ophoff D, Slaats MA, Boudewyns A, Glazemakers I, Van Hoorenbeeck K, Verhulst SL. Sleep disorders during childhood: a practical review. Eur J Pediatr. 2018;177(5):641-48.

34. Corrêa CC, Cavalheiro MG, Maximino LP, Weber SA. Obstructive sleep apnea and oral language disorders. Braz J Otorhinolaryngol. 2017;83(1):98-104.

35. Raphael KG, Santiago V, Lobbezoo F. Is bruxism a disorder or a behaviour? Rethinking the international consensus on defining and grading of bruxism. J Oral Rehabil. 2016;43(10):791-98.

\section{CONFLITO DE INTERESSES}

Os autores declaram não haver conflitos de interesse

\section{AUTOR PARA CORRESPONDÊNCIA}

\section{Camila de Castro Corrêa}

Professora do Centro Universitário Planalto do Distrito Federal (UNIPLAN) e da Universidade de Brasília (UnB) Fonoaudióloga (FOB-USP), Doutora em Bases Gerais da Cirurgia (FMB-UNESP)

Especialista em Motricidade Orofacial (CFFa), Aperfeiçoamento em MO (HC-FMRP-USP)

Certificada em Fonoaudiologia do Sono (Associação Brasileira do Sono)

Lattes: http://buscatextual.cnpq.br/buscatextual/visualiz acv.do?id=K4487727H0

E-mail: camila.ccorrea@hotmail.com

Submetido em 23/11/2020

Aceito em 14/12/2020 